\title{
Including four-gluon interactions into Landau and maximally-Abelian gauge Dyson-Schwinger studies
}

\author{
Valentin Mader* \\ U. Graz \\ E-mail: valentin.mader@uni-graz.at \\ Reinhard Alkofer \\ U. Graz \\ E-mail: reinhard.alkoferduni-graz.at
}

In Dyson-Schwinger studies of the Yang-Mills propagators the four-gluon interaction has been usually neglected due to the related technical difficulties with the associated two-loop terms and especially their renormalization. A possible scenario to renormalize these fully-dressed twoloop terms is presented. Preliminary results for the Landau gauge gluon propagator are shown. Implications for the gluon propagators in maximally Abelian gauge are discussed.

Xth Quark Confinement and the Hadron Spectrum

October 8-12, 2012

TUM Campus Garching, Munich, Germany

\footnotetext{
* Speaker.
} 


\section{Motivation}

Dyson-Schwinger equations (DSEs) provide a non-perturbative tool for studying quantum field theories. Being an infinite set of coupled equations for the $n$-point functions of the theory, they contain in principle all information about the observables of the underlying theory. In addition, since they are derived from the renormalized action, they are fully renormalized equations and, given a certain regularization and renormalization scheme, all divergences are absorbed in appropriately determined renormalization constants.

In practical calculations, however, truncations of the full infinite system to a finite subset of equations have to be performed. In general these truncations will interfere with the renormalization of DSEs and divergences will reappear. In addition an inappropriate choice of a regulator might also introduce divergences, as e.g., a hard cut-off in numerical calculations. Thus even though the full infinite set of DSEs might be fully renormalized, in practical calculations divergences have to be properly subtracted. Furthermore, perturbative renormalization is not sufficient due to the self-consistent nature of DSEs.

In Dyson-Schwinger studies of Landau gauge Yang-Mills propagators in four dimensions especially spurious quadratic divergences caused problems, since they are related to the breaking of gauge symmetry by the regularization/truncation scheme. Successful treatements were, e.g., identification and subtraction in the relevant tensor-components of the gluon self-energy [U, ㅁ] , and the construction of explicit subtraction terms within the integral kernels [B], 田, 可]. On the other hand, logarithmic divergences can be treated straightforwardly in a MOM-scheme.

In studies of the Landau gauge gluon propagator DSE truncations were chosen such that all terms containing a four-gluon interaction have been neglected, see, however, ref. [G]. A special role is played by the tadpole diagram: It only contributes a quadratically divergent constant, which is then removed in the renormalization process. The other terms with a four-gluon interaction are of two-loop order, the so-called sunset and squint diagrams. As the gluon-dressing function resulting from DSE studies is in the intermediate momentum region somewhat smaller than the ones obtained from lattice calculations or the Functional Renormalization group [1] it has been speculated that the two-loop terms might provide the missing contribution. Therefore it is desirable to include the sunset and the squint diagram into DSE studies of the Yang-Mills propagators propagators in Landau gauge.

Most non-perturbative studies of the Yang-Mills propagators have been done in Landau gauge. Another covariant gauge, where in the last years progress has been gained, is the Maximally

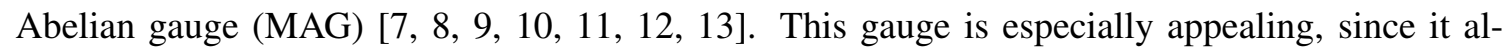
lows to study the dual superconductor picture of the Yang-Mills vacuum. In this picture, confinement is realized via a dual Meissner effect and color-electric fields are squeezed into flux-tubes by the screening property of the vacuum. This relates to the hypothesis of Abelian dominance [14]] which postulates a dominance of the abelian degrees of freedom over the non-abelian ones in the infrared. This hypothesis could also be confirmed in an infrared analysis of the untruncated Dyson-Schwinger equations in this gauge [W]. This investigation showed that the abelian (diagonal) gluons are enhanced in the infrared in contrast to the off-diagonal gluons and ghosts, which are infrared suppressed. Hereby, the infrared leading diagram in the gluon propagator DSE in MAG is the sunset and possibly the squint diagram. However, both, sunset and squint diagrams, include 


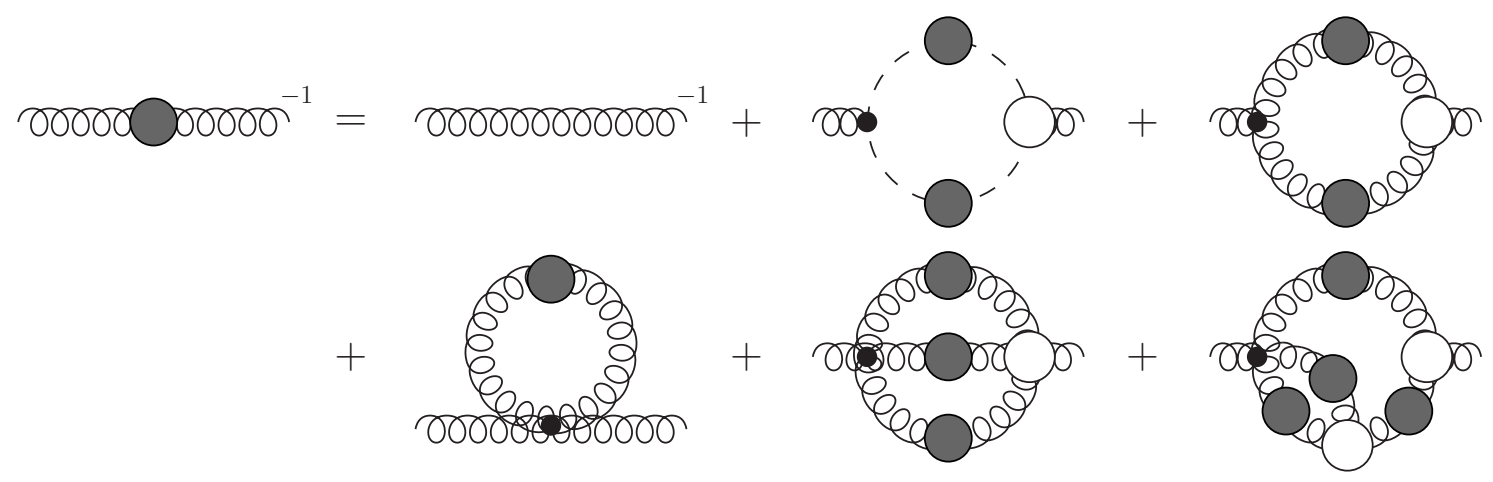

Figure 1: The Dyson-Schwinger equation of the gluon-propagator in Landau gauge.

four-point interactions.

In these proceedings we report on progress which has been gained in including the sunset diagram into Dyson-Schwinger studies of Yang-Mills theory in four dimensions. A main step is the identification and subtraction of overlapping quadratic divergences. We present results on the contribution of the sunset diagram to the gluon dressing function in Landau gauge and propose a possible minimal truncation of the propagator DSEs in the MAG.

\section{Dyson-Schwinger equations in Landau gauge}

The Dyson-Schwinger equation for the gluon propagator in Landau gauge is depicted in Fig.W Over the last decade a truncation scheme has evolved in which all terms containing four-gluon interactions were excluded. In addition the ghost-propagator DSE was taken into account, which left only two unknown Green-functions in the system, the ghost-gluon and three-gluon vertex, which were modeled. ${ }^{1}$ We want to start from this truncation with the vertex models as introduced in Ref. [3] and then add the sunset diagram to this system.

The presented truncation, and the usage of a hard momentum cut-off, introduce quadratic divergences. One possibility to deal with these divergences is to modify the integration kernel in the gluon-loop such that they cancel the quadratic divergences from the ghost-loop [B]]. The corresponding renormalization condition is the vanishing of the gluon pole mass, $Z_{m} m^{2}=0$, which holds for any order in perturbation theory ${ }^{2}$. The logarithmic divergencies are then subtracted in a MOM-scheme. When now including the sunset new overlapping quadratic divergences appear. Since there is no partner to cancel these divergences, we have to subtract the quadratic divergences within the diagram itself. To fulfill the corresponding renormalization condition we have to completely subtract these divergences without any additive constant. As it will be seen below, for a specific momentum partitioning we are able to construct a modified integration kernel which yield an integral without any quadratic divergences.

\footnotetext{
${ }^{1}$ See, however, the recent investigation [ㄷ]] in which a dynamic ghost-gluon vertex have been taken into account.

${ }^{2}$ Note that this is not in contradiction to the family of decoupling solutions [10, प17, ज] of Landau gauge DSEs which

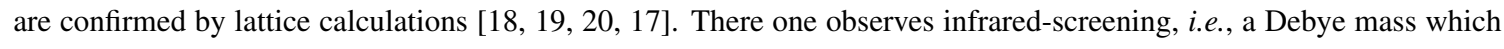
for the scaling solution would just be infinite.
} 


\section{The sunset diagram: Ultraviolet (UV) analysis}

The analytic expression of the sunset diagram in the DSE of the gluon propagator is given by

$$
\Pi_{\mu v \text { sunset }}^{a b}(k)=-Z_{4} \frac{1}{6} \int \vec{d}^{4} q_{1} \int \vec{d}^{4} q_{2} \Gamma_{\mu \rho \sigma \tau}^{(0) a r s t} \Gamma_{v \rho^{\prime} \sigma^{\prime} \tau^{\prime}}^{b r^{\prime} t^{\prime}}\left(-p, p_{1}, p_{2}, p_{3}\right) D_{\sigma \sigma^{\prime}}^{s s^{\prime}}\left(p_{1}\right) D_{\rho \rho^{\prime}}^{r r^{\prime}}\left(p_{2}\right) D_{\tau \tau^{\prime}}^{t t \prime^{\prime}}\left(p_{3}\right)
$$

with the bare and fully dressed four-gluon vertices, $\Gamma^{(0)}$ and $\Gamma$, resp., and the fully dressed gluonpropagator $D$. The $p_{i}$ denote the gluon momenta, and the $q_{i}$ some appropriately chosen loop momenta, see below. We have also used the short-hand notation $\int \vec{d}^{4} q=\int d^{4} q /(2 \pi)^{4}$. To proceed we have to assume a model for the dressed four-gluon vertex. In this study we assume a tree-level tensor structure mulitiplied with a scalar dressing function which will be modeled such that the correct $\mathrm{UV}$ and IR behavior is reproduced, $\Gamma_{v \alpha \beta \gamma}^{a b c d}\left(-p, p_{1}, p_{2}, p_{3}\right) \approx D_{\Gamma}\left(-p, p_{1}, p_{2}, p_{3}\right) \Gamma_{v \alpha \beta \gamma}^{(0) a b c d}$. Inserting also the definition for the gluon propagators $D_{\mu v}^{a b}(q)=\delta^{a b} T_{\mu \nu}(q) Z\left(q^{2}\right) / q^{2}$ with the transverse projector $T_{\mu v}(q)=\delta_{\mu v}-q_{\mu} q_{v} / q^{2}$ and projecting the equation with the generalized Brown-Pennington projector, $T_{\mu v}^{\zeta}(p)=\frac{1}{3}\left(\delta_{\mu v}-\zeta p_{\mu} p_{v} / p^{2}\right)$, and $\delta^{a b}$ one obtains

$$
\Pi_{\text {sunset }}^{\text {proj }}=-Z_{4} \frac{1}{6} g^{4} N_{c}^{2} \int d^{4} q_{1} \int d^{4} q_{2} \mathscr{T} D_{\Gamma}\left(-p, p_{1}, p_{2}, p_{3}\right) \frac{Z\left(p_{1}^{2}\right) Z\left(p_{2}^{2}\right) Z\left(p_{3}^{2}\right)}{p_{1}^{2} p_{2}^{2} p_{3}^{2}}
$$

with the tensor $\mathscr{T}$ being given by

$$
\begin{aligned}
\mathscr{T}:= & 45+9 z_{12} z_{13} z_{23}-\zeta\left(9+9\left(z_{01} z_{03} z_{13}+z_{01} z_{02} z_{12}+z_{02} z_{03} z_{23}\right)\right. \\
& +3\left(z_{01}^{2}+z_{02}^{2}+z_{03}^{2}+z_{12}^{2}+z_{13}^{2}+z_{23}^{2}-z_{01}^{2} z_{23}^{2}-z_{02}^{2} z_{13}^{2}-z_{03}^{2} z_{12}^{2}\right) \\
& \left.+3\left(z_{01} z_{02} z_{13} z_{23}+z_{01} z_{03} z_{12} z_{23}+z_{02} z_{03} z_{12} z_{13}\right)\right)
\end{aligned}
$$

and $z_{i j}:=p_{i} \cdot p_{j} / \sqrt{p_{i}^{2} p_{j}^{2}}$. Choosing now a specific momentum partitioning, $p_{1}=q_{1}, p_{2}=p+$ $q_{2}, p_{3}=-q_{1}-q_{2}$, and integrating out those angles which do not appear in the integrand we arrive at

$$
\begin{aligned}
\Pi_{\text {sunset }}^{\mathrm{proj}}=-\frac{Z_{4} g^{4} N_{c}^{2}}{3(2 \pi)^{6}} \int_{0}^{\Lambda} d q_{1} \int_{0}^{\Lambda} d q_{2} \int_{-1}^{1} d z_{1} \sqrt{1-z_{1}^{2}} \int_{-1}^{1} d z_{2} \sqrt{1-z_{2}^{2}} \int_{-1}^{1} d y \\
q_{1} q_{2}^{3} \mathscr{T} D_{\Gamma}\left(-p, q_{1}, p+q_{2},-q_{1}-q_{2}\right) \frac{Z\left(q_{1}^{2}\right) Z\left(\left(p+q_{2}\right)^{2}\right) Z\left(\left(q_{1}+q_{2}\right)^{2}\right)}{\left(p+q_{2}\right)^{2}\left(q_{1}+q_{2}\right)^{2}} .
\end{aligned}
$$

To gain insight into the UV behavior of Eq. (B.4) a method which has proven to be very useful in the UV analysis of DSEs in asymptotically free theories is the so-called y-max approximation [[1], []]. In the high-momentum regime the gluon dressing function behaves according to the perturbative logarithmic behavior. Due to this weak momentum dependence one can then safely neglect the dependence of the dressing functions on the angles. Since the cut-off can be choosen arbitrarily large the largest contributions to the UV behaviour will originate from the momentum region $\Lambda>q_{1}, q_{2}>p$. For the sunset additionally we have to assume that exceptional momenta will not contribute significantly to the integral which for the employed non-perturbative gluon propagators is justified due to their infrared (IR) suppression. Here we implement a scaling solution, which even vanishes for $p^{2} \rightarrow 0$. Under these assumptions one can integrate over the angles analytically 
and obtains

$\Pi_{\text {sunset }}^{\mathrm{y} m a x}=-\frac{Z_{4} g^{4} N_{c}^{2}}{3(2 \pi)^{6}} \int_{p}^{\Lambda} d q_{1} \int_{p}^{\Lambda} d q_{2} D_{\Gamma}\left(-p, q_{1}, p+q_{2},-q_{1}-q_{2}\right) Z\left(q_{1}^{2}\right) Z\left(\left(p+q_{2}\right)^{2}\right) Z\left(\left(q_{1}+q_{2}\right)^{2}\right) I_{\gtrless}$.

For $q_{1}>q_{2}$ the integrand $I_{\gtrless}$ is given by,

$$
I_{\gtrless}=-\frac{189 \pi^{2}}{32} \frac{q_{2}}{q_{1}}(\zeta-4)+\frac{9 \pi^{2}}{32} \frac{q_{2}^{3}}{q_{1}^{3}}(\zeta-4)-\frac{9 \pi^{2}}{32} \frac{p^{2} q_{2}}{q_{1}^{3}}(\zeta-1)
$$

and for $q_{2}>q_{1}$ one gets,

$$
\begin{aligned}
I_{\gtrless}=-\frac{189 \pi^{2}}{32} \frac{q_{1}}{q_{2}}(\zeta-4)+\frac{9 \pi^{2}}{32} & \frac{q_{1}^{3}}{q_{2}^{3}}(\zeta-4)-\frac{27 \pi^{2}}{32} \frac{p^{2} q_{1}}{q_{2}^{3}} \\
& +\frac{3 \pi^{2}}{16} \frac{p^{2} q_{1}^{3}}{q_{2}^{5}}(6+\zeta)-\zeta \frac{15 \pi^{2}}{32}\left(\frac{p^{4} q_{1}^{3}}{q_{2}^{7}}+\frac{p^{2} q_{1}^{5}}{q_{2}^{7}}-\frac{p^{4} q_{1}^{5}}{q_{2}^{9}}\right) .
\end{aligned}
$$

The explicit expressions Eqs. (B.6) allow to identify the quadratic divergences and construct a subtraction term such that upon integration the quadratic divergences are canceled for both cases. This procedure provides a "regulated" tensor structure,

$$
\widetilde{\mathscr{T}}=\mathscr{T}-(\zeta-4)\left(-\frac{225}{16}+\frac{9}{4}\left(z_{12}+3 z_{01}\right)\right)
$$

If we replace $\mathscr{T}$ in Eq. (B.4) by $\widetilde{\mathscr{T}}$, no quadratic divergences will appear.

A few comments are in order here. First note that the quadratic divergences come with a factor $\zeta-4$. This means that the quadratic divergences of the sunset only contribute to the $\delta_{\mu v}$-component of the gluon polarization tensor as is expected from general considerations [四]. Second, in the past two-loop diagrams were not included due to the complications provided by their overlapping divergences. However, using the regulated tensor structure Eq. (B.]) the quadratic divergences are cancelled directly. As the explicit form of this subtraction term depends on the particularly chosen momentum partitioning this has become possible due to the specific choice taken, and whether a similar subtraction is possible for general momentum partitionings remains to be clarified.

\section{A model for the four-gluon vertex dressing}

The unknown required input into Eq. (B.4) is the dressing function of the four-gluon vertex. While the UV behavior of this function is known from perturbation theory only little is known when one of the momenta becomes small. ${ }^{3}$ In refs. [R], [2] ] an infrared behavior of the four-gluon vertex consistent with the scaling solutions of the DSEs has been determined. Under consideration of the available information we model the vertex dressing function as a product of the propagator dressing functions:

$$
D_{\Gamma}\left(-p, p_{1}, p_{2}, p_{3}\right)=\frac{1}{Z_{4}} \frac{\left[G\left(p_{1}^{2}\right) G\left(p_{2}^{2}\right)\right]^{\alpha}}{Z\left(p_{3}^{2}\right)\left[Z\left(p_{1}^{2}\right) Z\left(p_{2}^{2}\right)\right]^{1-\beta}},
$$

\footnotetext{
${ }^{3}$ To our knowledge there is no lattice study of this object.
} 

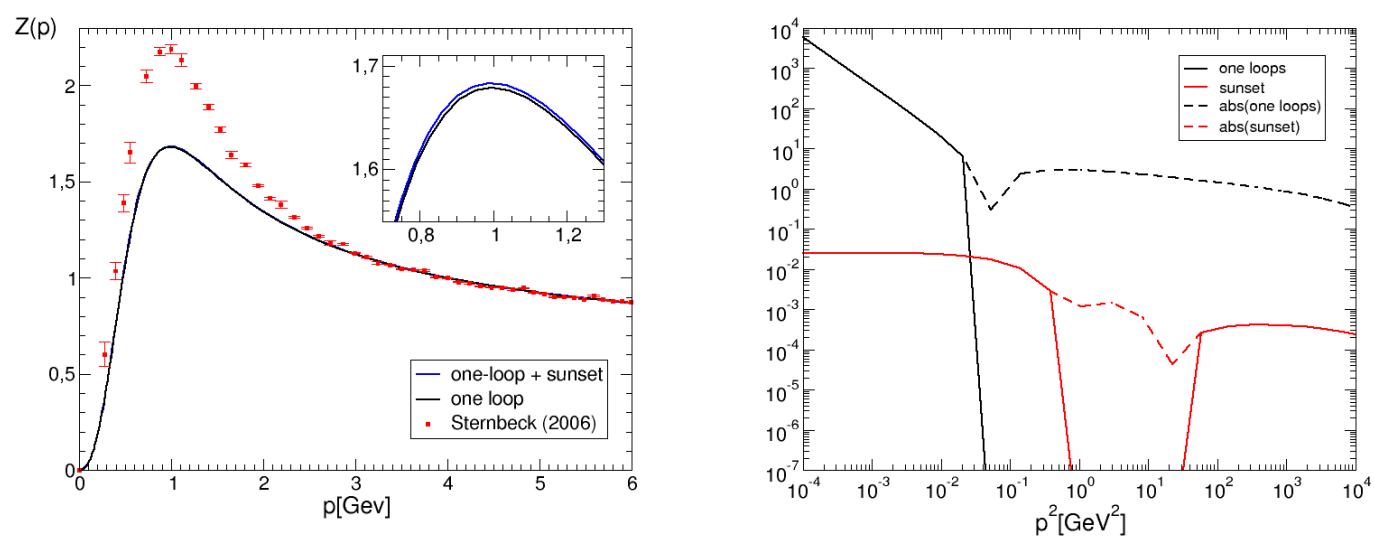

Figure 2: Left: The Landau gauge gluon dressing function with (blue) and without (black) contributions from the sunset diagram compared to lattice data of Ref. [एष]. Right: Contributions of the one-loop diagrams and the sunset after subtracting quadratic divergencies.

where we introduced two parameters $\alpha$ and $\beta$ which are determined by the UV and IR behaviour. For high energies the dressing function are required to obey a logarythmic scaling $D_{\Gamma} \sim(\log x)^{-\gamma+2 \delta}$ with the anomalous dimensions of the gluon propagator, $\gamma=-\frac{13}{22}$, and the ghost propagator $\delta=$ $-\frac{9}{44}$, respectively. To be consistent with the scaling solution in the IR we require $D_{\Gamma} \sim(x)^{-4 \kappa}$ with the IR scaling exponent $\kappa \approx 0.59535$ [23], 2]]. Thus we can determine the parameters to $\alpha=-4 \delta$ and $\beta=\frac{1}{2}(1-4 \delta)$.

In this first preliminary investigation this model has been chosen mainly due to technical reasons. Besides the missing Bose symmetry the different treatment of the propagators even within the sunset diagram calls for improvement. As we divide out the dressing function with argument $p_{3}$ this propagator becomes undressed which allows us to integrate over two of the three angles analytically. This saves a substantial amount of computing time. We will improve on this shortcoming via transporting these numerical calculations onto GPUs along the lines of Ref. [24]. This should enable us to calculate all angular integrals numerically which is a prerequisite to keep Bose symmetry in the employed four-gluon-vertex model.

For the numerical calculation one still needs to choose a partitioning of the two radial integrals. In addition, there exist integrable divergences for $q_{1}-q_{2} \rightarrow 0$ which have to be treated seperately. Details will be published elsewhere.

\section{Results and outlook}

Within the approximations described above we included the sunset diagram into a Landau gauge DSE study. As one can see from Fig.】 the influence of the sunset diagram is astonishingly small. This is in contrast to expectations that the two-loop terms should contribute significantly in the mid-momentum regime. Even our preliminary results provide compelling evidence that the sunset diagram is completely unimportant in the gluon propagator DSE. At this point two remarks are in order: First, the precise form of the three-guon vertex in the one-gluon-loop term is very 


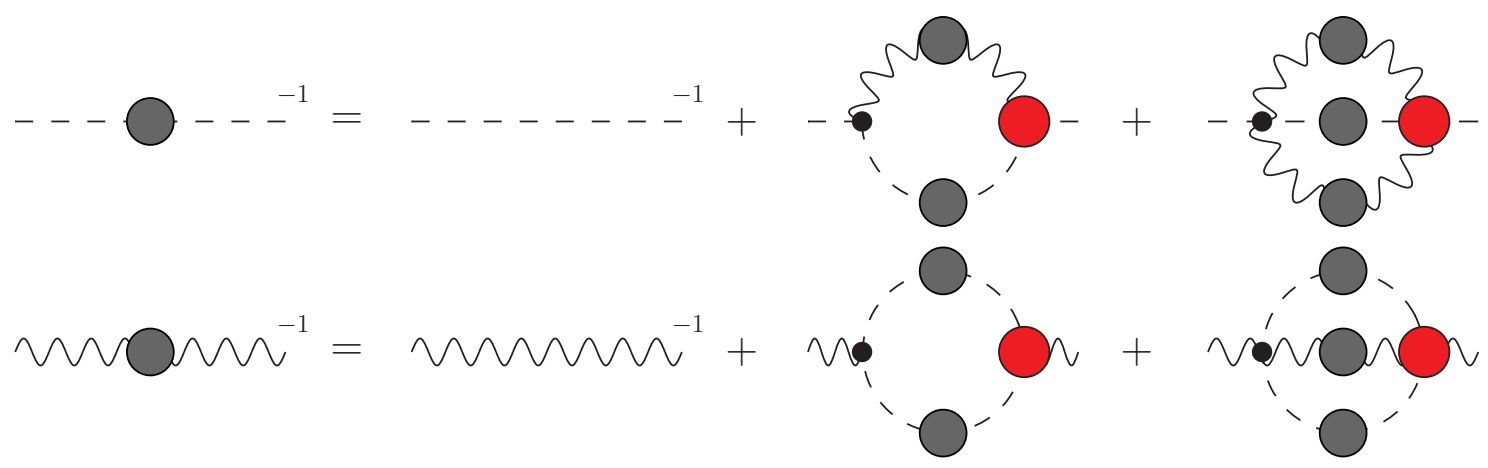

Figure 3: The proposed equations for ghosts and diagonal gluon in the MAG.

important as can be seen from very recently obtained results [대] also presented on this conference. ${ }^{4}$ An improvement of the three-gluon vertex model, especially the inclusion of a sign flip at small momenta, leads to an enhancement of the gluon dressing function in the mid-momentum regime. Second, preliminary results on including the squint diagram in the gluon propagator DSE [26] point towards a small but still sizeable contribution. Here the use of a Bose symmetric four-gluon dressing function is then mandatory to allow for definite conclusions.

\section{Implications on the Maximally Abelian gauge}

As mentioned an IR analysis of the Yang-Mills propagator DSEs and Renormalization Group Equations in the Maximally Abelian gauge showed [W] that the sunset diagram is an IR leading term. We propose here a minimal set of coupled equations for the propagators of ghost and diagonal gluon in the Maximally Abelian gauge which is depicted in Fig. B. It is the minimal truncation of the corresponding DSEs which contains the leading UV and IR behaviour. The corresponding oneloop terms give the correct UV behavior [9], where as the sunset diagrams give the IR behavior [W]. Also there are only two vertices which have to be modeled to close the system, the three-point $(A \bar{c} c)$ - and the four point $(A A \bar{c} c)$-vertex. Again we assume a tree-level structure for these vertices dressed with a scalar function which reproduces the correct UV and IR behaviour of these Green functions. The quadratic divergences can be subtracted along the same line as presented above: A $y$-max approximation allows for the construction of a regularized tensor structure in the integral kernels. The logarthmic divergences are then treated in a MOM-scheme. Numerical studies of this set of equations as a starting point of an investigation of the Yang-Mills propagators in the Maximally Abelian gauge are in progress.

\section{Acknowledgements}

We are grateful to the organizers of the Xth Quark Confinement and the Hadron Spectrum conference for all their efforts which made this extraordinary event possible.

We thank A. Sternbeck for providing us lattice results. VM is funded by the Austrian Science

\footnotetext{
${ }^{4}$ See, however, also Refs. [[2]] for a corresponding discussion of the importance of the one-gluon-loop term.
} 
Fund, FWF, through the Doctoral Program on Hadrons in Vacuum, Nuclei, and Stars (FWF DK W1203-N16).

\section{References}

[1] N. Brown and M. R. Pennington, Phys. Rev. D 39 (1989) 2723.

[2] D. Atkinson and J. C. R. Bloch, Phys. Rev. D 58 (1998) 094036 [hep-ph/9712459].

[3] C. S. Fischer and R. Alkofer, Phys. Rev. D 67 (2003) 094020 [hep-ph/0301094].

[4] C. S. Fischer, PhD thesis 2003 [hep-ph/0304233].

[5] C. S. Fischer, A. Maas and J. M. Pawlowski, Annals Phys. 324 (2009) 2408 [arXiv:0810.1987 [hep-ph]].

[6] J. C. R. Bloch, Phys. Rev. D 64 (2001) 116011 [hep-ph/0106031].

[7] T. Shinohara, K. I. Kondo and T. Murakami, Nucl. Phys. Proc. Suppl. 129 (2004) 748 [hep-lat/0309164].

[8] V. G. Bornyakov et al., Phys. Lett. B 559 (2003) 214 [hep-lat/0302002].

[9] J. A. Gracey, JHEP 0504 (2005) 012 [hep-th/0504051].

[10] M. A. L. Capri et al., Braz. J. Phys. 37 (2007) 591 [hep-th/0603167].

[11] M. Q. Huber, K. Schwenzer and R. Alkofer, Eur. Phys. J. C 68 (2010) 581 [arXiv:0904.1873 [hep-th]]; PoS FACESQCD (2010) 001 [arXiv:1103.0236 [hep-th]].

[12] R. Alkofer, M. Q. Huber, V. Mader and A. Windisch, PoS QCD -TNT-II (2011) 003 [arXiv:1112.6173 [hep-th]].

[13] S. Gongyo, T. Iritani and H. Suganuma, Phys. Rev. D 86 (2012) 094018 [arXiv:1207.4377 [hep-lat]].

[14] Z. F. Ezawa and A. Iwazaki, Phys. Rev. D 25 (1982) 2681.

[15] M. Q. Huber and L. von Smekal, arXiv:1211.6092 [hep-th]; PoS CONFINEMENTX (2013) 062 [arXiv:1301.3080 [hep-th]].

[16] A. C. Aguilar, D. Binosi and J. Papavassiliou, Phys. Rev. D 78 (2008) 025010 [arXiv:0802.1870 [hep-ph]].

[17] P. Boucaud et al., Few Body Syst. 53 (2012) 387 [arXiv:1109.1936 [hep-ph]].

[18] A. Sternbeck, PhD thesis, 2006 [hep-lat/0609016].

[19] A. Cucchieri and T. Mendes, Phys. Rev. D 78 (2008) 094503 [arXiv:0804.2371 [hep-lat]].

[20] I. L. Bogolubskyet al., Phys. Lett. B 676 (2009) 69 [arXiv:0901.0736 [hep-lat]].

[21] C. S. Fischer and J. M. Pawlowski, Phys. Rev. D 75 (2007) 025012 [hep-th/0609009].

[22] C. Kellermann and C. S. Fischer, Phys. Rev. D 78 (2008) 025015 [arXiv:0801.2697 [hep-ph]].

[23] C. Lerche and L. von Smekal, Phys. Rev. D 65 (2002) 125006 [hep-ph/0202194];

D. Zwanziger, Phys. Rev. D 65 (2002) 094039 [hep-th/0109224].

[24] M. Hopfer, R. Alkofer and G. Haase, Comput. Phys. Comm. 184 (2013) 1183 [arXiv:1206.1779 [hep-ph]].

[25] A. Maas, J. Wambach, B. Gruter and R. Alkofer, Eur. Phys. J. C 37 (2004) 335 [hep-ph/0408074]; Eur. Phys. J. C 42 (2005) 93 [hep-ph/0504019].

[26] M. Hopfer, V. Mader and R. Alkofer, work in progress. 